\title{
Boundary Flows in general Coset Theories
}

\author{
Changrim Ahn * \\ Institut für Theoretishe Physik, Freie Universität Berlin \\ Arnimallee 14, Berlin D-14195, Germany \\ and \\ Chaiho Rim \\ Department of Physics \\ Chonbuk National University, Chonju 561-756, Korea
}

\begin{abstract}
In this paper we study the boundary effects for off-critical integrable field theories which have close analogs with integrable lattice models. Our models are the $S U(2)_{k} \otimes S U(2)_{l} / S U(2)_{k+l}$ coset conformal field theories perturbed by integrable boundary and bulk operators. The boundary interactions are encoded into the boundary reflection matrix. Using the TBA method, we verify the flows of the conformal BCs by computing the boundary entropies. These flows of the BCs have direct interpretations for the fusion RSOS lattice models. For super CFTs $(k=2)$ we show that these flows are possible only for the Neveu-Schwarz sector and are consistent with the lattice results. The models we considered cover a wide class of integrable models. In particular, we show how the impurity spin is screened by electrons for the $k$-channel Kondo model by taking $l \rightarrow \infty$ limit. We also study the problem using an independent method based on the boundary roaming TBA. Our numerical results are consistent with the boundary CFTs and RSOS TBA analysis.
\end{abstract}

EWHA-TH-9801

SNUTP-98-043

APCTP-98-012

*Permanent Address: Department of Physics, Ewha Womans University, Seoul 120-750, Korea 


\section{Introduction}

In the study of the two-dimensional quantum field theories and statistical models, the conformal field theories (CFT) [1] have provided both theoretical frameworks and powerful tools. This applies to off-critical models, not to mention critical ones. Various off-critical quantities can be related to those of CFTs. The two-body scattering amplitude ( $S$-matrix) of a perturbed CFT [2] can reproduce the central charge and identify the perturbing field using the thermodynamic Bethe ansatz (TBA) [3] and provide a proof that most integrable quantum field theories are CFTs perturbed by some relevant operator along which the theory extends away from criticality. Two-dimensional lattice models with Boltzmann weights satisyfing the Yang-Baxter equation (YBE) also show these properties. For example, the order parameters such as local height probabilities (LHP) of the restricted solid-on-solid (RSOS) models can generate critical exponents like the central charges of minimal CFTs [- [4] and are related to the characters of the CFTs [5].

Such relations between critical and off-critical theories in the presence of boundary are main question investigated in this paper. There have been many interesting progresses in the quantum field theory in the space-time with boundary. In paricular, existence of the boundary in $(1+1)$ dimensions changes conserved quantities, symmetries and integrability and requires new formulations for both CFTs and integrable off-critical models. To maintain infinite dimensional conformal symmetries, the CFTs should introduce new quantities, the conformal boundary states, which have one-to-one correspondence with conformal boundary conditions (CBC) [6].

Off-critical models which can be represented by a symbolic action

$$
\mathcal{A}=\mathcal{A}_{\mathrm{CFT}+\mathrm{CBC}}+\Lambda \int d^{2} x \Phi+\lambda \int d t \phi_{B},
$$

can maintain the integrability if one choose the perturbing fields carefully. For example, it is shown in [8] that the minimal CFTs perturbed by the least relevant bulk and boundary primary fields can be integrable. Once the integrability is implemented, we need new quantities called the boundary reflection amplitudes ( $R$-matrices) which are the probability amplitudes for a particle to scatter off from the boundary. The boundary YBE and bootstrap arguments can determine these amplitudes completley with a given $S$-matrix, the bulk scattering amplitude, as an input [9, 8]. As the bulk TBA based on the $S$-matrix give the CFT data underlying in the perturbed CFTs such as central charges, the $R$-matrix can be used to find the information on the boundary conditions. This is our main question investigated in this paper. 
TBA analysis with the boundary should be a natural method for this purpose. Recently, TBA for the perturbed minimal CFTs with certain BCs has been used to find the boundary entropies and corresponding CBCs [10]. With certain $R$-matrices, the CBCs flow into each other which are consistent with independent study based on the roaming TBA [11]. This result seems very interesting since it can provide a general method to understand the relations of the $R$-matrix and CFTs data in (四). In this paper, we generalize this result on the minimal models to general coset CFTs [12] which include the superconformal series and $Z_{k}$ parafermionic algebras [13]. The minimal CFTs are only the first of many infinite series of these CFTs. One can obtain a variety of off-critical integrable models associated with these CFTs which include many interesting ones.

These questions are not merely of academic interests. They can be directly related to physical quantities measurable in experiment. Recent progress have shown that these theoretical tools can be very efficient for quantitative understanding of non-perturbative aspects of boundary problems. For example, boundary CFTs [6] have been applied to multi-channel Kondo models [14] and integrable field theories to transport phenomena in quantum impurity problems [15]. The boundary entropies associated with CBCs are one of main physical quantities in these computations at UV and IR fixed points. Our methods for the general coset theories with certain limits are used to understand the flows between the CBCs quantitatively. Our results will be of use to estabilish the boundary TBA method to compute the boundary entropy and to understand boundary behaviours in the intermediate boundary scale for a wide class of integrable models including the multi-channel Kondo model.

This paper is orgarnized as follows. In sect.2, we define the general CFTs and associated lattice models. We derive the boundary entropies for the CBCs of these CFTs following standard procedure. The boundary perturbations of these CFTs are understood in terms of massless scattering matrices in sect.3. These $S$-matrices are used to compute the boundary entropies using the TBA. In sect.4, we compute the flows of the boundary entropies using an independent analysis, namely, boundary roaming TBA. The boundary roaming TBA of super CFTs are obtained by analytic continuation of that of the supersymmetric sinh-Gordon (SShG) model. Similar analysis is proposed for the parafermionic models. Numerical analysis shows that the results are consistent with those obtained from the boundary CFTs and RSOS TBA. We conclude with some discussions in sect.5. 


\section{2 coset CFT with boundary}

\section{1 coset CFTs}

Most rational CFTs can be expressed as coset CFTs [12]. In particular, general $S U(2)$ coset CFTs $\mathcal{M}(k, l)$ represented schematically as

$$
\mathcal{M}(k, l)=\frac{S U(2)_{k} \otimes S U(2)_{l}}{S U(2)_{k+l}}
$$

with central charges

$$
c(k, l)=\frac{3 k}{k+2}\left[1-\frac{2(k+2)}{(l+2)(k+l+2)}\right]
$$

include many important and frequently used CFTs. Here, $S U(2)_{k}$ is the level $k$ Wess-Zumino-Witten (WZW) model with Kac-Moody algebra as an extended conformal symmetry [16]. Besides the minimal CFTs $\mathbb{1}] \mathcal{M}(1, l)$, there are many other series with some extended symmetries which will be of our main concern. Our convention is that the first index $k$ of $\mathcal{M}(k, l)$ denotes the extended symmetries.

In particular, the superconformal theories $\mathcal{M}(2, l)$ with $c=c(2, l)$ with the primary fields $\Phi_{(r, s)} 1 \leq r \leq p-1,1 \leq s \leq p+1$ [17. We will use often $p$ for $l+2$ throughout the paper. Here, $\Phi_{(r, s)}$ is identified with $\Phi_{(p-r, p+2-s)}$. The super CFTs and their representations can be classified into two sectors, the Neveu-Schwarz (NS) and Ramond (R) sectors, which are selected by antiperiodic or periodic boundary conditions on fermionic fields, respectively. In the above notations, the primary field $\Phi_{(r, s)}$ belongs to the (NS) or (R) sectors depending on $r-s$ even or odd integers.

In general the coset CFTs $\mathcal{M}(k, l)(k$ fixed and $l=1,2, \ldots)$ are extended CFTs with $Z_{k}$ parafermion currents 18. The characters of the coset theories are defined as branching functions

$$
\chi_{t}^{[k]}(\tau, z) \chi_{r}^{[l]}(\tau, z)=\sum_{s=1}^{k+l+1} B_{r, s}^{t}(\tau) \chi_{s}^{[k+l]}(\tau, z),
$$

where $\chi_{r}^{[l]}$ is the character of the highest weight $r(r=1, \ldots, l+1)$ for the $S U(2)_{l}$ WZW model. The primary fields for the coset CFTs, $\Phi_{r, s}^{t}$, have three weights which take values in

$$
\begin{aligned}
& t=|(r-s) \bmod 2 k|+1, \quad 1 \leq t \leq k+1 \\
& 1 \leq r \leq l+1, \quad 1 \leq s \leq k+l+1
\end{aligned}
$$


The index $t$ stands for the sectors of the extended symmetries. For example, $t=1,3$ corresponds to the (NS) and $t=2$ to the $(\mathrm{R})$ sector of the supersymmetry $(k=2)$. Since $\mathcal{M}(k, l) \equiv \mathcal{M}(l, k)$, one can have two different realizations for the CFTs. $\mathcal{M}(2,3)$ can be the third CFT of the super CFTs or the second CFT of the $Z_{3}$ parafermion theory by rearranging the conformal modules.

One of the fundamental quantities in CFTs is the modular $S$-matrix for $B_{r, s}^{t}(\tau)$ which can be obtained by tranforming $\tau \rightarrow-1 / \tau$ in the above expression [7] and using that of $S U(2)_{l}$ WZW model. The results aref

$$
S_{(r, s)}^{\left[t, t^{\prime}\right]} \underset{\left(r^{\prime}, s^{\prime}\right)}{ }=\sqrt{\frac{8}{(k+2) p(p+k)}} \sin \frac{\pi t t^{\prime}}{k+2} \sin \frac{\pi r r^{\prime}}{p} \sin \frac{\pi s s^{\prime}}{p+k} .
$$

The modular $S$-matrix can be simplified by choosing special sectors as

$$
S_{(r, s)}^{\left(r^{\prime}, s^{\prime}\right)}=\text { const. } \sin \frac{\pi r r^{\prime}}{p} \sin \frac{\pi s s^{\prime}}{p+k}
$$

where the constant factor is not of our concern since it will be cancelled out in the quantities of interest.

For the super CFTs, the $S$-matrices of the two sectors are in general complicated except the (NS)-(NS) $S$-matrix [19 given by

$$
S_{(r s)}^{\left(r^{\prime} s^{\prime}\right)}=\frac{4}{\sqrt{p(p+2)}} \sin \frac{\pi r r^{\prime}}{p} \sin \frac{\pi s s^{\prime}}{p+2} .
$$

For the reason explained below, we will restrict our analysis to the (NS) sector.

\section{2 boundary conditions}

CFTs can make sense in two dimensions with a boundary only with welldefined CBCs classified by Cardy [6]. With the boundary conditions on both sides of the strip $\alpha, \beta$, the partition function $Z_{\alpha \beta}$ can be expressed as

$$
Z_{\alpha \beta}(q)=\sum_{i} n_{\alpha \beta}^{i} \chi_{i}(\tau),
$$

${ }^{1}$ Strictly speaking, the characters of the coset CFTs are linear combinations of the branching functions belonging to the same sector ( $t=1$ and 3 in the super CFTs). The modular $S$-matrix for the characters will be modified except some sectors like the (NS) where this complicacy disappears. 
where $n_{\alpha \beta}^{i}$ denotes the number of times that the irreducible representation $i$ occurs under the $\mathrm{BC} \alpha \beta$. Using the modular transformation $\tau \rightarrow-1 / \tau$, one can reexpress this as

$$
Z_{\alpha \beta}(q)=\sum_{j}\langle\alpha \mid j\rangle\langle j \mid \beta\rangle \chi_{j}(-1 / \tau)
$$

from which one drives the Cardy equation,

$$
\sum_{i} S_{i}^{j} n_{\alpha \beta}^{i}=\langle\alpha \mid j\rangle\langle j \mid \beta\rangle .
$$

The state $|\alpha\rangle$ satisfying this equation defines the CBC. It is found that for each primary field $\Phi_{l}$ there corresponds a $\mathrm{CBC}|\tilde{l}\rangle$ which is defined in such a way that the partition function with this $\mathrm{CBC}$ on one side is identified with the character of $\Phi_{l}$, namely,

$$
Z_{\tilde{0} \tilde{h}_{i}}=\chi_{i}(q) .
$$

This boundary state is expressed as a linear combination of the primary states of the CFT,

$$
\left|\tilde{h}_{i}\right\rangle=\sum_{j} \frac{S_{i}^{j}}{\sqrt{S_{0}^{j}}}|j\rangle .
$$

Since $|0\rangle$ defines the ground state of the CFT, $\langle 0 \mid \tilde{l}\rangle$ defines the ground degeneracy of the boundary state. This boundary degeneracy $g$ is given by

$$
g_{i} \equiv\left\langle 0 \mid \tilde{h}_{i}\right\rangle=\frac{S_{i}^{0}}{\sqrt{S_{0}^{0}}},
$$

and the boundary entropy, defined by $s_{\mathrm{B}}=\log g$, can be completely determined by the modular $S$-matrix elements.

For the general coset CFTs, one can use Eq.(5) to get the boundary degeneracies for a simplest sector,

$$
g_{(r, s)}^{[k, l]}=\text { const. } \frac{\sin \frac{\pi r}{l+2} \sin \frac{\pi s}{k+l+2}}{\sqrt{\sin \frac{\pi}{l+2} \sin \frac{\pi}{k+l+2}}} .
$$

In particular, degeneracies for the boundary states $(1, s)$ and $(r, 1)$ are given by

$$
\begin{aligned}
g_{(1, s)}^{[k, l]} & =\operatorname{const} .\left(\frac{\sin \frac{\pi}{l+2}}{\sin \frac{\pi}{k+l+2}}\right)^{1 / 2} \sin \frac{\pi s}{k+l+2} \\
g_{(r, 1)}^{[k, l]} & =\operatorname{const} .\left(\frac{\sin \frac{\pi}{k+l+2}}{\sin \frac{\pi}{l+2}}\right)^{1 / 2} \sin \frac{\pi r}{l+2} .
\end{aligned}
$$


Using (4) and (10) one can find the boundary degeneracies for the (NS) sector $\tilde{h}_{(1, s)}$ and $\tilde{h}_{(r, 1)}$ of the super CFTs as follows $(r, s$ odd):

$$
\begin{aligned}
& g_{(1, s)}^{[2, p-2]}=\left(\frac{16}{p(p+2)}\right)^{1 / 4}\left(\frac{\sin \frac{\pi}{p}}{\sin \frac{\pi}{p+2}}\right)^{1 / 2} \sin \frac{\pi s}{p+2} \\
& g_{(r, 1)}^{[2, p-2]}=\left(\frac{16}{p(p+2)}\right)^{1 / 4}\left(\frac{\sin \frac{\pi}{p+2}}{\sin \frac{\pi}{p}}\right)^{1 / 2} \sin \frac{\pi r}{p} .
\end{aligned}
$$

\subsection{Fusion RSOS lattice model}

For later purposes, it is useful to have lattice model realizations for the general coset CFTs. The generalizations of the original RSOS model [4 have the Boltzmann weights $W(a, b, c, d)$ defined by four heights at four corners of a square, each taking values in the $A_{k+l+1}$-Dynkin diagram, where two adjacent heights are subject to the following conditions [5]

$$
\begin{aligned}
& a=1,2, \ldots, k+l+1, \\
& a-b=-k,-k+2, \ldots, k-2, k \\
& (a+b-k) / 2=0,1, \ldots, l+1 .
\end{aligned}
$$

From the $Z_{2}$ automorphism of the Dynkin diagram, the model is equivalenet under the simultaneous change of $a \rightarrow k+l+2-a$. The LHP $P(a / b, c)$, the probability for a height to be $a$ under the boundary heights to have $(b, c)$, have been computed and related to the branching function of $\mathcal{M}(k, l)$ in the regime III,

$$
B_{d, a}^{e}, \quad \text { with } \quad e=\frac{b-c+k}{2}+1, \quad d=\frac{b+c-k}{2} .
$$

Notice that the range of $d, e$ with Eq.(15) is consistent with Eqs.(3, 3).

It is interesting to express the boundary states in terms of the RSOS lattice models. It is found in 200 that the partition function of the RSOS lattice model $(k=1)$ with boundary heights fixed as $(a / b, c)$ in Fig.(1) is given by

$$
Z(a / b, c)=\chi_{d, a}
$$

with $d$ is $\inf (b, c)$, smaller one of $b, c$. From Eq.(8), one can conclude that $(1 / b, c) \mathrm{BC}$ of the RSOS lattice model corresponds to the CBC $\left|\tilde{h}_{(d, 1)}\right\rangle$. Another $\mathrm{BC}$ we will consider is the case of free $b$ while $a=1$ and $c$ are fixed, which corresponds to the $\mathrm{CBC}\left|\tilde{h}_{(1, c)}\right\rangle$. 


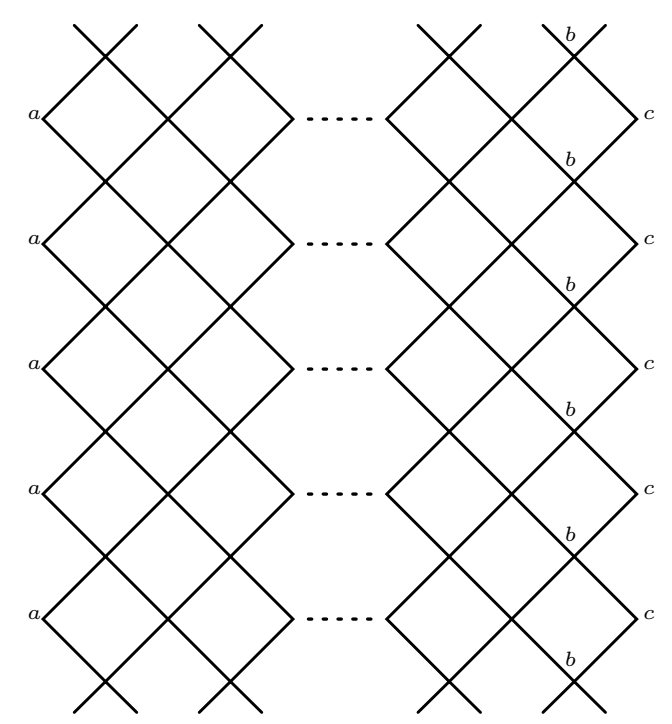

Figure 1: RSOS lattice with $(a / b, c)$ BC

Now let us consider the boundary conditions for the general $k \geq 2$ cases, namely the 'fusion' RSOS models. If the argument of the conformal transformation of the strip to the annulus in 20] are still valid for the fusion RSOS lattice, the transfer matrix with fixed $\mathrm{BC}$ on the strip will be related to the corner transfer matrix. This means that the partition function $Z(a / b, c)$ on the strip is related to the LHP $P(a / b, c)$, hence to the the branching function in Eq.(16). Therefore, we can conjecture that analogous results can hold for the fusion models; namely, $(1 / b, c)$ BC of the fusion model corresponds to the $\mathrm{CBC}\left|\tilde{h}_{(d, 1)}^{e}\right\rangle$ with $d$, e given above. Using the invariance under $c \rightarrow k+p-c$, we will restrict the boundary heights to $1 \leq b, c \leq(k+p) / 2$.

From (15), $b$ can take three values, namely $c-2, c+2, c$ for $k=2$. Plugging into (16), one can find that $b=c \pm 2$ and $b=c$ correspond to the $(\mathrm{NS})$ and $(\mathrm{R})$ sectors, respectively. One can also see that $c-a$ should be even since the differences of two neighboring heights should be even. This means the $\mathrm{BC}(1 / b, c)$ makes sense only for odd $b, c$. Because Eq.(3) with $r=d$ and $s=a$ gives $t=1,3$, the boundary states should always belong to the (NS) sector with $b=c \pm 2$. Similarly $(1 / c)$ BC with odd $c$ is identified with the $\mathrm{CBC}\left|\tilde{h}_{(1, c)}^{\mathrm{NS}}\right\rangle$. We summarize as follows (d,c are odd integers):

$$
\begin{array}{rlrl}
(1 / c \pm 2, c) & \rightarrow\left|\tilde{h}_{(d, 1)}^{\mathrm{NS}}\right\rangle & \text { with } & 1 \leq d=[(c-1) \pm 1] \leq \frac{p}{2}-1 \\
(1 / c) & \rightarrow\left|\tilde{h}_{(1, c)}^{\mathrm{NS}}\right\rangle \quad \text { with } & 1 \leq c \leq \frac{p}{2}+1
\end{array}
$$




\section{Massless Boundary Scattering}

\subsection{Bulk RSOS TBA}

It has been claimed years ago that the minimal CFTs perturbed by the least relevant operator are integrable and can be described by RSOS scattering theories [21] $S_{\mathrm{RSOS}(k)}(\theta)$, the RSOS $S$-matrix whose quantum group parame-

ter is given by $q=-e^{i \pi /(k+2)}$. For the perturbed general coset CFTs, similar results have been obtained where the particles carry two sets of RSOS spins, namely, $\left|K_{a, b}(\theta)\right\rangle \otimes\left|K_{c, d}(\theta)\right\rangle$ with $S$-matrices 22]

$$
S(\theta)=S_{\mathrm{RSOS}(k)}(\theta) \otimes S_{\mathrm{RSOS}(l)}(\theta) .
$$

The first set of RSOS spins $(a, b)$ acting on the first $S$-matrix is considered as the index for internal symmetries such as supersymmetry $(k=2)$. These particles, 'kinks', are obtained by restricting multi-soliton Hilbert space when the quantum group parameter $q$ is a root of unity. These massive theories correspond to the perturbed CFTs with negative coefficients. We denote this by $\mathcal{M} A^{(-)}(k, l)$.

If the coefficients of the perturbing operator are positive, the perturbed CFTs will flow between two fixed points [24]. These flows of the central charges have been reproduced using the above $S$-matrix in the thermodynamic Bethe ansatz (TBA) analysis by changing only the dispersion relation to $E= \pm P= \pm M e^{ \pm \theta}$ 225] which means the left-moving (-) and right-moving $(+)$ massless particles. $M$ is a mass scale which is connected with the dimensionful perturbing parameter $\Lambda$. These theories, denoted by $\mathcal{M} A^{(+)}(k, l)$, with $S_{L L}$ and $S_{R R}$ given by Eq.(19) and with appropriate $S_{L R}$ are interpolating two adjacent CFT series in the following way [26]:

$$
\mathcal{M}(k, l) \quad \rightarrow \quad \mathcal{M}(k, l-k)
$$

Notice that there are more than one sequences of the flows within CFTs with fixed $k \geq 2$.

Furthermore, it has been claimed in [27] that in the vanishing limit of $\Lambda$, one can still preserve the massless kink spectrum along with the RSOS $S$ matrices. Since the perturbed CFTs in the limit of vanishing perturbations are obviously the CFTs, these scattering theories can describe the CFTs. Only difference from the $\mathcal{M} A^{(+)}(k, l)$ is that $S_{L R}=1$, i.e. trivial scattering between $L$ and $R$-movers. All these theories are invariant under $k \leftrightarrow l$.

Non-pertubative results can be obtained by the TBA. It is very complicated to derive the TBA equations for the RSOS $S$-matrices and is not of 
(a)
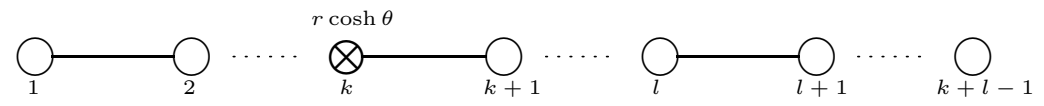

(b)
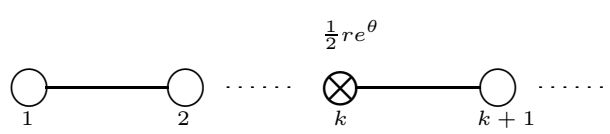

$\frac{1}{2} r e^{-\theta}$

(c)
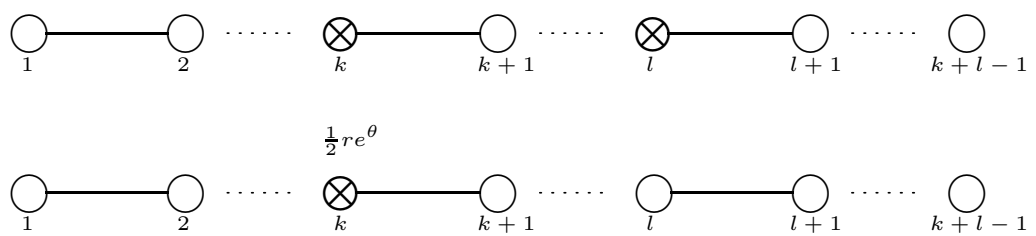

$\frac{1}{2} r e^{\theta}$

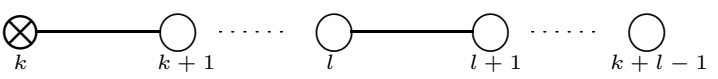

Figure 2: TBA diagrams (a) $\mathcal{M} A^{(-)}(k, l)$ (b) $\mathcal{M} A^{(+)}(k, l)$ (c) $\mathcal{M}(k, l)$

our concern. For detailed derivations, see [28]. Instead, we sketch briefly the conceptual aspects only which will be useful to understand the boundary cases. With nondiagonal $S$-matrices, one needs to diagonalize the transfer matrices arising in the periodic BC. The eigenvalues depends on the particle rapidities as well as the 'magnonic' mode which, in turn, satisfies some constraint equation. To define this constraint, one needs to introduce another mode and so on. For $\operatorname{RSOS}(k)$, one needs $k-1$ massless magnonic modes. By interpreting these modes as massless particles and the constraints as the periodic $\mathrm{BC}$, one can transform the nondiagonal problem into the that of diagonal scattering theories. The rest of the derivation is straightforwardly standard. Since only the first magnon rapidity will enter to define the eigenvalues, the massive particle scatters with the first magnon and the first with the second etc.

For the general cases with $S$-matrix (19), the transfer matrix will be also the tensor product form and the eigenvalues are products of two factors which have two sets of magnons $(k-1$ for $\operatorname{RSOS}(k)$ and $l-1 \operatorname{RSOS}(l))$. In the effective diagonal TBA, the massive particle scatters with two first magnons and the first with the second for a given factor and so on. This is represented in the TBA diagram of Fig.2(a) TBA for $\mathcal{M} A^{(+)}(k, l)$ and $\mathcal{M}(k, l)$ are conjectured similarly and represented in Fig.2(b) and (c). Here, the index $k$ is the smaller of $k, l$. The exchange of $k$ and $l$ does not change the TBA.

Explicit TBA equations are expressed as follows:

$$
\epsilon_{a}(\theta)=\nu_{a}(\theta)-\sum_{b=1}^{k+l-1} l_{a b} \varphi * L_{b}(\theta), \quad a=1, \ldots, k+l-1,
$$


where

$$
\begin{aligned}
\varphi(\theta) & =\frac{1}{\cosh \theta} \\
L_{b}(\theta) & =\log \left(1+e^{-\epsilon_{b}(\theta)}\right) \\
f * g(\theta) & =\int \frac{d \theta^{\prime}}{2 \pi} f\left(\theta-\theta^{\prime}\right) g\left(\theta^{\prime}\right),
\end{aligned}
$$

and the source terms $\nu_{a}(\theta)$ are given by

$$
\begin{aligned}
& \nu_{a}(\theta)=\delta_{a k} r \cosh \theta \text { for } \mathcal{M} A^{(-)}(k, l) \\
& \nu_{a}(\theta)=\delta_{a k} r \frac{e^{\theta}}{2}+\delta_{a l} r \frac{e^{-\theta}}{2} \text { for } \mathcal{M} A^{(+)}(k, l) \\
& \nu_{a}(\theta)=\delta_{a k} r \frac{e^{\theta}}{2} \text { for } \mathcal{M}(k, l)
\end{aligned}
$$

where a dimensionless parameter $r$ definded by $M / T$ with temperature $T$ interpolates the UV $(r \rightarrow 0)$ and IR $(r \rightarrow \infty)$ limits. $l_{a b}$ is the incidence matrix whose elements are 1 if two nodes $a, b$ are connected in the TBA diagram Fig.(2) or 0 otherwise.

In the UV and IR limits, $\epsilon_{a}$ 's only at $\theta= \pm \infty, 0$ are important and can be determined by simple algebraic equations. Two of the massless TBA have the same solutions in the UV limit 28, 26]

$$
\begin{aligned}
1+e^{-\epsilon_{a}(-\infty)} & =\left[\frac{\sin \frac{\pi(a+1)}{k+l+2}}{\sin \frac{\pi}{k+l+2}}\right]^{2}, \quad 1 \leq a \leq k+l-1 \\
1+e^{-\epsilon_{a}(\infty)} & =\left[\frac{\sin \frac{\pi(a+1-k)}{l+2}}{\sin \frac{\pi}{l+2}}\right]^{2}, \quad k \leq a \leq k+l-1 \\
1+e^{-\epsilon_{a}(\infty)} & =\left[\frac{\sin \frac{\pi(a+1)}{k+2}}{\sin \frac{\pi}{k+2}}\right]^{2}, \quad 1 \leq a \leq k,
\end{aligned}
$$

while the IR behaviours are all different. $\mathcal{M} A^{(-)}(k, l)$ becomes massive with $e^{-\epsilon_{a}}=0 . \mathcal{M}(k, l)$ remains same and independent of $r$ while $\mathcal{M} A^{(+)}(k, l)$ generates the flows (20) where the $\epsilon_{a}$ 's are given as above with replacing $l \rightarrow l-k$.

\subsection{Boundary RSOS TBA}

Now we introduce the boundary. The formal action is in the form of Eq.(1). The perturbed CFTs can be well-defined only after specifying the CBCs. 
Once the integrability is maintained by specific BCs, the boundary $R$-matrix, obtained by the boundary YBE, can be used in the boundary TBA to compute the entropies [29]. For diagonal $S$ - and $R$-matrices,

$$
\begin{aligned}
\log \left\langle B_{\alpha} \mid 0\right\rangle\left\langle 0 \mid B_{\beta}\right\rangle & =\int_{-\infty}^{\infty} \frac{d \theta}{2 \pi} \kappa_{\alpha \beta}(\theta) \log \left(1+e^{-\epsilon(\theta)}\right)+\text { const. } \\
\epsilon(\theta) & =\frac{1}{2} r e^{\theta}+\phi * L(\theta) \\
\kappa_{\alpha \beta}(\theta) & =\frac{1}{i} \frac{d}{d \theta} \log \left[R_{\alpha}\left(\theta-\theta_{B_{\alpha}}\right) R_{\beta}\left(\theta-\theta_{B_{\beta}}\right)\right]
\end{aligned}
$$

where 'boundary rapidity' $\theta_{\mathrm{B}}$ is defined by $m_{B}=M e^{\theta_{\mathrm{B}}}$ where the boundary mass scale $m_{B}$ is a certain power of $\lambda$ in (1). The UV (IR) limit is $\theta_{B} \rightarrow$ $-\infty(\infty)$.

The simplest example is $\mathcal{M}(1,1)$, namely the Ising model with $R$ given by

$$
R\left(\theta-\theta_{B}\right)=-i \tanh \left[\frac{1}{2}\left(\theta-\theta_{B}\right)-\frac{i \pi}{4}\right]
$$

which produces the boundary entropy $s_{\mathrm{B}}=\log \langle 0 \mid B\rangle$ as

$$
s_{\mathrm{B}}=\int_{-\infty}^{\infty} \frac{d \theta}{2 \pi} \frac{\log \left(1+e^{-\epsilon(\theta)}\right)}{\cosh \left(\theta-\theta_{B}\right)}+\text { const. }
$$

This gives the correct flow of the boundary degeneracy, $g_{\mathrm{UV}} / g_{\mathrm{IR}}=\sqrt{2}$.

Generalizing this result to the $\operatorname{RSOS}(k \geq 2)$ theory is nontrivial. The authors in 10 claimed that the boundary perturbation of the minimal CFT with the $\mathrm{CBC}\left|\tilde{h}_{(1, a)}\right\rangle$ is the quantum group reduction of the massless limit of the boundary sine-Gordon model with the anisotropic spin- $j$ Kondo interaction at boundary. Therefore, the boundary $R$-matrices of the boundary $\operatorname{RSOS}(k)$ theory are given by the RSOS version of the $R$-matrix of the Kondo model given in [30]:

$$
\begin{aligned}
& R(\theta)=1, \quad a=1 \\
& R(\theta)=-i \tanh \left(\frac{\theta-\theta_{\mathrm{B}}}{2}-\frac{i \pi}{4}\right), \quad a=2 \\
& R(\theta)=R^{1 / 2,(a-2) / 2}\left(\theta-\theta_{\mathrm{B}}\right), \quad 3 \leq a \leq \frac{p+1}{2},
\end{aligned}
$$

with $a=2 j+1$. Notice $j=1 / 2(a=2)$ where the boundary spin is fixed at spin- $1 / 2$ gives the same $R$ as the Ising model. Based on these $R$-matrices, 
the boundary entropy for the CBC $\left|\tilde{h}_{(1, a)}\right\rangle$ has been conjectured as

$$
s_{\mathrm{B}}^{(a)}=\int \frac{d \theta}{2 \pi} \frac{\log \left(1+e^{-\epsilon_{a-1}(\theta)}\right)}{\cosh \left(\theta-\theta_{\mathrm{B}}\right)},
$$

where $\epsilon_{a}$ 's are determined by usual RSOS bulk TBA. With solutions of the bulk TBA, the ratios of the boundary degeneracies become

$$
\frac{g_{\mathrm{UV}}^{(a)}}{g_{\mathrm{IR}}^{(a)}}=\frac{1+e^{-\epsilon_{a-1}(-\infty)}}{1+e^{-\epsilon_{a-1}(\infty)}} .
$$

These results can be a guideline for the general coset CFTs. Considering the bulk $S$-matrix (19), one can look for the $R$-matrix in the form of

$$
R(\theta)=R_{\mathrm{RSOS}(k)}(\theta) \otimes R_{\mathrm{RSOS}(l)}(\theta) .
$$

The first factor is related to the internal symmetry. The fractional supersymmetry is defined in the $S$-matrix of the $\operatorname{RSOS}(k)$ [21]. In the bulk conformal limit $\Lambda \rightarrow 0$, this symmetry will remain as extended conformal symmetries generated by the $Z_{k}$ parafermion. Even in the presence of boundary, we require that the CFTs should preserve these extended symmetries along with the conformal ones. Among many solutions [31] for the boundary $R$-matrix of the $\operatorname{RSOS}(k)$ model, no one can preserve the conserved currrents of fractional spin except the trivial case of $R \propto \mathbf{1}$. This is consistent with the results in [32] where the $R$-matrix of massless particles with topological charges corresponding to the Dirichlet $\mathrm{BC}$ is $\mathbf{1}$. We will use the $R$-matrix (26) for the second $R$. To summarize, our conjecture for the $R$-matrix of the CBC $\left|\tilde{h}_{(1, a)}\right\rangle$ is $\mathbf{1} \otimes R^{1 / 2,(a-2) / 2}$.

The boundary TBA can be derived similarly. While Boundary part will be the same as before, Eqs.(27,28), since the reflection amplitudes do not change, bulk part will be described by TBA of $\mathcal{M}(k, l)$. Let's consider for the case of $a>k$ first. Plugging Eqs.(23,24) into (28), one gets

$$
\frac{g_{\mathrm{UV}}^{(a)}}{g_{\mathrm{IR}}^{(a)}}=\frac{\sin \frac{\pi a}{k+l+2}}{\sin \frac{\pi}{k+l+2}} \frac{\sin \frac{\pi}{l+2}}{\sin \frac{\pi(a-k)}{l+2}} .
$$

Comparing (29) with Eqs.(11, 12), we find that the boundary reflection matrices generate the flows

$$
\left|\tilde{h}_{(1, a)}\right\rangle \rightarrow\left|\tilde{h}_{(a-k, 1)}\right\rangle .
$$


This means the UV CBC $\left|\tilde{h}_{(1, a)}\right\rangle$ of the perturbed boundary CFTs $\mathcal{M}(k, l)$ changes to $\left|\tilde{h}_{(a-k, 1)}\right\rangle$ in the IR $(a \geq k+1)$.

It will be more instructive to intepret this result in the fusion RSOS lattice model language. For $k+1 \leq a \leq(k+l+2) / 2$, Eq.(30) means simply the flow from $(1 / a) \mathrm{BC}$ to $(1 / a-k, a)$. For $(k+l+2) / 2 \leq a \leq$ $k+l+1$ the $\mathrm{CBC}\left|\tilde{h}_{(1, a)}\right\rangle$ and $\left|\tilde{h}_{(a-k, 1)}\right\rangle$ correspond to the lattice BCs $\left(1 / a^{\prime}\right)$ and $\left(1 / a^{\prime}+k, a^{\prime}\right)$ with $a^{\prime}=k+l+2-a$ due to the $Z_{2}$ symmetry. According to Eq.(16), these boundary states are identified with the CBC $\left|\tilde{h}_{\left(1, a^{\prime}\right)}\right\rangle$ and $\left|\tilde{h}_{\left(a^{\prime}, 1\right)}\right\rangle$, respectively. Therefore, the boundary TBA results give two flows with $k+1 \leq a \leq(k+l+2) / 2$,

$$
\begin{aligned}
& \left|\tilde{h}_{(1, a)}\right\rangle \rightarrow\left|\tilde{h}_{(a-k, 1)}\right\rangle \quad \text { or } \quad(1 / a) \rightarrow(1 / a-k, a) \\
& \left|\tilde{h}_{(1, a)}\right\rangle \rightarrow\left|\tilde{h}_{(a, 1)}\right\rangle \quad \text { or } \quad(1 / a) \rightarrow(1 / a+k, a) .
\end{aligned}
$$

Our results reproduce [10 for $k=1$ where it is claimed that these flows are associated with the signs of $\lambda$ in (1). Since $\lambda$ is only free parameter, this conclusion should be also true for general cases.

For the case of $a \leq k$, the boundary degeneracies can be computed from $(23,25)$. The result is

$$
\frac{g_{\mathrm{UV}}^{(a)}}{g_{\mathrm{IR}}^{(a)}}=\frac{\sin \frac{\pi a}{k+l+2}}{\sin \frac{\pi}{k+l+2}} \frac{\sin \frac{\pi}{k+2}}{\sin \frac{\pi a}{k+2}}=\frac{g_{(1, a)}^{[l, k]}}{g_{(a, 1)}^{[l, k]}},
$$

where we used Eqs.(11,12) at the last equality. Notice that the indices $k$ and $l$ are switched. This means the the $\mathrm{BC}$ flow for the $Z_{l}$, not $Z_{k}$, parafermion CFTs;

$$
\left|\tilde{h}_{(1, a)}\right\rangle \rightarrow\left|\tilde{h}_{(a, 1)}\right\rangle .
$$

For the lattice model, this is the flow $(1 / a) \rightarrow(1 / a+k, a)$ and with (32) $a$ is now extended to $1 \leq a \leq(k+l+2) / 2$.

Our model covers a wide range of integrable models for each $k$ and $l$. In particular, the result for $l \rightarrow \infty$ limit is interesting and has also physical applicability. The coset CFT in this limit becomes the level- $k$ WZW model with boundary interaction. This is the multi-channel Kondo model of the spin current considered in [14]. For $a=2 j+1>k$, Eq.(29) gives

$$
\frac{g_{\mathrm{UV}}^{(a)}}{g_{\mathrm{IR}}^{(a)}}=\frac{a}{a-k}, \quad a>k,
$$

which means that the spin degeneracy of $2 j+1$ at the UV limit flows into $2(j-k / 2)+1$ in the IR. This is nothing but the screening effect of the impurity 
spin by $k$-channel electrons in the underscreend case which can be obtained by 'fusion hypothesis' [14. The case of $2 j+1 \leq k$ is more interesting. Eq.(33) gives

$$
\frac{g_{\mathrm{UV}}^{(a)}}{g_{\mathrm{IR}}^{(a)}}=a \cdot \frac{\sin \frac{\pi}{k+2}}{\sin \frac{\pi a}{k+2}}
$$

This is exactly the result for the overscreened Kondo model previously obtained by the Bethe ansatz and boundary CFT results.

Our TBA result can be used to understand the flows quantitatively. We show the numerical result of the boundary entropies for several boundary spins $(k=3$ and $l=4)$ as the boundary scale changes in Fig.3. This graph illustrates nonperturbatively the ' $g$-theorem' that the boundary entropy always decreases as the system goes from UV to IR [14].

\subsection{Massless flows in the Bulk and Boundary}

We considered so far the case of $\Lambda=0, \lambda \neq 0$ which shows only the boundary flows. Natural extension will be the simultaneous flow of the bulk and boundary. Due to nontrivial scattering between the right- and left-movers, the bulk TBA is given by (21). For the boundary entropy, an educated guess is

$$
s_{\mathrm{B}}^{(a)}=\frac{1}{2} \int_{-\infty}^{\infty} \frac{d \theta}{2 \pi}\left[\frac{\log \left(1+e^{-\epsilon_{a-1}(\theta)}\right)}{\cosh \left(\theta-\theta_{\mathrm{B}}\right)}+\frac{\log \left(1+e^{-\epsilon_{k+l+1-a}(\theta)}\right)}{\cosh \left(\theta+\theta_{\mathrm{B}}\right)}+\text { const. }\right] .
$$

This is the same conjecture used in 10.

Analysis of this boundary TBA is a little more complicated. In the bulk UV limit $r=M / T \rightarrow 0$, we define $\theta_{\mathrm{B}}=\bar{\theta}+\beta_{\mathrm{B}}$ with $\bar{\theta} \rightarrow \infty$ so that $r e^{\bar{\theta}}$ is finite. Then redefine the rapidity as $\theta= \pm(\bar{\theta}+\beta)$ for $R(L)$-movers. For $R$ movers, the source term at node $l$ vanishes reducing $\nu_{a}$ 's to those of $\mathcal{M}(k, l)$ and the second term in (34) also vanishes and the boundary entropy is the half of $\mathcal{M}(k, l)$. For $L$-movers, the source term at node $k$ and the first term in (34) vanish. The resulting TBA and boundary entropy are the same as those by $R$-movers using the obvious symmetry of the TBA diagram and the solutions (23,24) under $k \leftrightarrow l$. These two contributions cancel the half in front of the entropy and gives the same formula as before. This result is expected since the two TBA systems are equivalent at $U V$.

Now consider the opposite limit, i.e. the bulk IR limit $M / T \rightarrow \infty$. Since $r \rightarrow \infty$, we redefine the rapidities differently, namely, $\theta_{\mathrm{B}}=-\bar{\theta}+\beta_{\mathrm{B}}$ and $\theta= \pm(\bar{\theta}-\beta)$ with $\bar{\theta} \rightarrow \infty$. For $R$-movers, the source term at node $k$ 
becomes infinite and the TBA diagram for $\mathcal{M} A^{(+)}(k, l)$ is cut at $k$. The remaining diagram is that of $\mathcal{M}(k, l-k)$ and the first term in (34) vanishes. With similar result for the $L$-movers, one gets the boundary TBA system with $l \rightarrow l-k$. Therefore, we get the boundary flows for the IR CFT $\mathcal{M} A^{(+)}(k, l)$.

Fixing the boundary scale $m_{B}$ and varying the bulk scale shows another interesting behaviour. For example, we consider (11) with CBC $\left|\tilde{h}_{(1, a)}\right\rangle$ and $\lambda=0$. In the bulk UV limit, the boundary degeneracy is given as before by

$$
g_{\mathrm{UV}}^{(a)}=\text { const. }\left(\frac{\sin \frac{\pi a}{k+l+2}}{\sin \frac{\pi}{k+l+2}}\right) .
$$

In the bulk IR limit with $r \rightarrow \infty$, the source terms diverge and the $k$ nodes from each end of the TBA diagram should be removed. The solutions can be obtained from (23) with replacing $a \rightarrow a-k$ and $k+l+2 \rightarrow l-k+2$ and the boundary entropy becomes

$$
g_{\mathrm{IR}}^{(a)}=\text { const. }\left(\frac{\sin \frac{\pi(a-k)}{l-k+2}}{\sin \frac{\pi}{l-k+2}}\right) .
$$

Using Eqs.(11, 12), one can confirm that these are the flows

$$
\left|\tilde{h}_{(1, a)}^{[k, l]}\right\rangle \rightarrow\left|\tilde{h}_{(a-k, 1)}^{[k, l-k]}\right\rangle .
$$

\section{Roaming on Boundary}

\section{1 super Roaming TBA}

Roaming model [11] is obtained by taking analytic continuation of the coupling constant of the sinh-Gordon model which generates all the minimal CFTs in one equation. As suggested in [33, this model can be used to describe the correlation functions via form factors of all the minimal series and their perturbations. Its application to the boundary problem is also tested in [10]. The supersymmetric version of this model is a nondiagonal theory and its bulk TBA has been derived using 'the free fermion condition' in [34].

The boundary reflection amplitude of the SShG model has been studied in [36]. Its boundary TBA for the $R$-matrix preserving the supersymmetry is found [37] as

$$
\begin{aligned}
& \epsilon_{1}(\theta)=r \cosh \theta-\varphi * L_{2}(\theta) \\
& \epsilon_{2}(\theta)=-\varphi * L_{1}(\theta),
\end{aligned}
$$


with the kernel

$$
\varphi(\theta)=\frac{4 \cosh \theta \sin (\pi \alpha)}{\cosh 2 \theta-\cos (2 \pi \alpha)}
$$

The boundary entropy is given by

$$
s_{B}=\frac{1}{4 \pi} \int d \theta \kappa(\theta) \log \left(1+e^{-\epsilon_{1}(\theta)}\right)
$$

where $\kappa(\theta)=-i \partial_{\theta} \log R$. By suppressing unnecessary parameters, $\kappa(\theta)$ is given by

$$
\kappa(\theta)=\frac{2 \cosh f \cosh \theta}{\cosh ^{2} \theta+\sinh ^{2} f}
$$

where $f$ is a dimensionless parameter determined by the mass scale of the bulk theory and the dimensionful parameter in the boundary potential. This is the same as the one appears in the roaming for the minimal model.

By taking an analytic continuation of the SShG coupling constant,

$$
\pi \alpha=\frac{\pi}{2} \pm \theta_{0} i \quad \text { with } \quad \theta_{0}>>1
$$

we obtain the roaming TBA with new kernel

$$
\varphi(\theta)=\frac{1}{\cosh \left(\theta-\theta_{0}\right)}+\frac{1}{\cosh \left(\theta+\theta_{0}\right)} .
$$

Our derivation of the bulk roaming TBA is exactly the one conjectured in [35] and generates the superconformal series with Witten index 1. The boundary entropies for super CFTs can be obtained numerically using (35) which are plotted in Fig.4 for $p=4,6,8$ Analyzing this figure, we can confirm that these values at the plateaus are consistent with $s_{B}=\log g$ with $g$ given in Eqs.(13, 14). We can find that there are two types of boundary flows, all in the $(\mathrm{NS})$ sector $(p=4 n$ or $4 n+2)$ :

$$
\begin{aligned}
& \left|\tilde{h}_{1, s}\right\rangle \rightarrow\left|\tilde{h}_{s-2,1}\right\rangle \text { for } \quad s=3,5, \ldots, 2 n+1, \\
& \left|\tilde{h}_{1, s}\right\rangle \rightarrow\left|\tilde{h}_{s, 1}\right\rangle \text { for } \quad s=3,5, \ldots, 2 n+1 .
\end{aligned}
$$

These are consistent with Eqs.(17,18) based on the boundary CFTs and with Eqs.(31,32) based on the RSOS TBA analysis. 


\section{2 boundary roaming for general coset}

Bulk roaming TBA equations for the general coset CFTs are conjectured in [38] as follows (We will consider only the $S U(2)$ coset CFTs.):

$$
\begin{aligned}
\epsilon^{(i)}(\theta) & =\nu^{(i)}(\theta)-\varphi_{-} * L^{(i-1)}(\theta)-\varphi_{+} * L^{(i+1)}(\theta), \\
\nu^{(i)}(\theta) & =\frac{1}{2}\left(\delta_{i, 0} r e^{-\theta}+\delta_{i, s} r e^{\theta}\right), \quad i=0, \ldots, k-1
\end{aligned}
$$

where the index is defined cyclic $i \equiv i+k$ and $\varphi_{ \pm}(\theta)=1 / \cosh \left(\theta \pm \theta_{0}\right)$ and $s=0, \ldots, k-1$. Without knowing any $S$-matrix interpretation of these equations, we can rely on our previous experience to conjecture the boundary roaming TBAs. Our conjecture is that the bulk part is the same as before and the boundary entropy is given by Eq. 35 with $\epsilon_{1}$ replaced by $\epsilon^{(0)}$. The bulk TBA is claimed to generate the roaming

$$
\ldots \rightarrow \mathcal{M}(k, 2 k+s) \rightarrow \mathcal{M}(k, k+s) \rightarrow \mathcal{M}(k, s)
$$

Our interest is the boundary entropy generated by the general roaming TBA. For example, we study numerically the boundary entropies for $k=3$ and $s=0$ and plot the result in Fig.5. From this, we conclude the following BC flows:

$$
\begin{aligned}
& \left|\tilde{h}_{1, s}\right\rangle \rightarrow\left|\tilde{h}_{s-3,1}\right\rangle \text { for } \quad s=4,7, \ldots, 3 n+1, \\
& \left|\tilde{h}_{1, s}\right\rangle \rightarrow\left|\tilde{h}_{s, 1}\right\rangle \text { for } \quad s=4,7, \ldots, 3 n+1 .
\end{aligned}
$$

Again, this result is consistent with (31,32) based on the boundary CFTs and RSOS scattering theories.

In Fig.4 and Fig.5, the second type flows $(1, s) \rightarrow(s, 1)$ are 'inverted' flows in the sense that the boundary entropies increase as the scale decreases. While this is not forbidden by the $g$-theorem since we are dealing with theories with complex coupling constant, hence complex dimensions, the origin is mysterious considering the roaming flows satisfy the 'c-theorem' faithfully.

\section{Conclusion}

In this paper, we have investigated a wide class of massless scattering theories originated as perturbed coset CFTs. The boundary scattering amplitudes are used to find the boundary entropies through the boundary TBA methods. Our key result is to verify the flows of the BCs both for the boundary CFTs 
and for the fusion lattice models. These results are cross-checked with the extended roaming TBAs.

Several points are not clarified. First of all, we have considered only the (NS) sector of the super CFTs and the corresponding results make sense only in that sector. For the fusion lattice model, it is shown that this is only possible case. The lattice realization of the $(\mathrm{R})$ sector and the analysis based on the boundary CFT and TBA remain to be resolved. Even for the (NS), we have considered only special BCs, namely $(r, 1)$ and $(1, s)$, equivalently $(1 / a)$ and $(1 / b, c)$ for the lattice model. The $R$-matrix we used describes the flows within this subset of BCs. Similarly, for $k>2$, we considered only special sector and special type of the BCs. It would be very interesting to find more general reflection matrices which can generate flows between other BCs.

We considered a wide class of integrable models, 'an integrable zoo' [22]. Putting a boundary for the zoo is a quite interesting project. In particular, various interesting results on the boundary can be obtained by taking various limits. The cases of $k=4, \infty$ with $l \rightarrow \infty$ give the bulk CFT with $c=$ 2,3 , which can be realized with two and three free bosons. The boundary behaviour of these theories may be interesting for string theory formulation.

Good agreement between the two results based on the RSOS and roaming TBAs suggest that the roaming limit of the super sinh-Gordon model with simple $S$-matrix can be useful to compute off-shell quantities such as correlators for more physically relevant models, such as multi-channel Kondo models.

\section{Acknowledgment}

We thank F. Lesage for explaining his work and CA thanks A. Fring for interesting conversations and Alexander von Humbodt foundation for financial support. This work is supported in part by KOSEF 961-0201-006-2 and BSRI 97-2427 (CA) and BSRI 97-2434 (CR) and by a grant from KOSEF through CTP/SNU. We also thank APCTP for sponsoring our program.

\section{Figure Caption}

- Fig.3: Flows of the boundary entropy as boundary scale changes for $k=3, l=4\left(s_{B}\right.$ vs. $\left.\theta_{B}\right)$ for various boundary states $a$. 
- Fig.4: Roaming of the boundary entropies for $k=2, s=0$ with $\theta_{0}=40$ $\left(f\right.$ vs. $\left.s_{B}\right)$.

1. $p=4$ :

(a) $\left|\tilde{h}_{1,3}\right\rangle$ to $\left|\tilde{h}_{1,1}\right\rangle(0.693 . . \rightarrow 0$.

2. $p=6$ :

(a) $\left|\tilde{h}_{1,3}\right\rangle$ to $\left|\tilde{h}_{1,1}\right\rangle(0.881 . . \rightarrow 0$.)

(b) $\left|\tilde{h}_{1,3}\right\rangle$ to $\left|\tilde{h}_{3,1}\right\rangle(0.881 . . \rightarrow 0.693 .$.

3. $p=8$ :

(a) $\left|\tilde{h}_{1,5}\right\rangle$ to $\left|\tilde{h}_{3,1}\right\rangle(1.173 . . \rightarrow 0.881 .),.\left|\tilde{h}_{1,3}\right\rangle$ to $\left|\tilde{h}_{1,1}\right\rangle(.962 . . \rightarrow 0$.

(b) $\left|\tilde{h}_{1,3}\right\rangle$ to $\left|\tilde{h}_{3,1}\right\rangle(.962 . . \rightarrow 0.881 .$.

- Fig.5: Roaming of boundary entropies for $k=3, s=0$ with $\theta_{0}=50(f$ vs. $\left.s_{\mathrm{B}}\right)$.

1. $p=5$ :

(a) $\left|\tilde{h}_{1,4}\right\rangle$ to $\left|\tilde{h}_{1,1}\right\rangle(0.9605 . . \rightarrow 0$.

2. $p=8$ :

(a) $\left|\tilde{h}_{1,4}\right\rangle$ to $\left|\tilde{h}_{1,1}\right\rangle(1.172 . . \rightarrow 0$.)

(b) $\left|\tilde{h}_{1,4}\right\rangle$ to $\left|\tilde{h}_{4,1}\right\rangle(1.172 . . \rightarrow 0.96054 .$.

3. $p=11$ :
(a) $\left|\tilde{h}_{1,7}\right\rangle$ to $\left|\tilde{h}_{4,1}\right\rangle(1.502 . . \rightarrow 1.172 .),.\left|\tilde{h}_{1,4}\right\rangle$ to $\left|\tilde{h}_{1,1}\right\rangle(1.256 . . \rightarrow 0$.
(b) $\left|\tilde{h}_{1,4}\right\rangle$ to $\left|\tilde{h}_{4,1}\right\rangle(1.256 . . \rightarrow 1.172 .$.

\section{References}

[1] A.A. Belavin, A.M. Polyakov and A.B. Zamolodchikov, Nucl. Phys. B241 (1984) 333.

[2] A.B. Zamolodchikov, Int. Journ. Mod. Phys. A4 (1989) 4235.

[3] Al. B. Zamolodchikov, Nucl. Phys. B342 (1990) 695.

[4] G. E. Andrews, R. J. Baxter and P. J. Forester, J. Stat. Phys. 35 (1984) 193. 
[5] E. Date, M. Jimbo, T. Miwa and M. Okado, Phys. Rev. B35 (1987) 2105.

[6] J.L. Cardy, Nucl. Phys. B240 (1984) 514.

[7] E. Date, M. Jimbo, T. Miwa and M. Okado, Nucl. Phys. B290 [FS20] (1987) 231.

[8] S.Goshal and A.B.Zamolodchikov, Int. J. Mod. Phys. A9 (1994) 3841.

[9] I.V.Cherednik, Teor. Mat. Fiz. 61 (1984) 35; E.K.Sklyanin, J. Phys. A21 (1988) 2375; A. Fring and R. Koberle, Nucl. Phys. B421 (1994) 159 .

[10] F. Lesage, H. Saleur and P. Simonetti, "Boundary flows in minimal models", hep-th/9802061.

[11] Al. B. Zamolodchikov, "Resonance factorized scattering and roaming trajectories", prepreint ENS-LPS-335, 1991.

[12] P. Goddard, A. Kent and D. Olive, Phys. Lett. B152 (1985) 88.

[13] D. Kastor, E. Martinec, and Z. Qiu, Phys. Lett. B200 (1988) 434; J. Bagger, D. Nemeschansky, and S. Yankielowicz, Phys. Rev. Lett. 60 (1988) 389; F. Ravanini, Mod. Phys. Lett. A3 (1988) 397.

[14] I. Affleck and A. W. W. Ludwig, Phys. Rev. Lett. 67 (1991) 161; Nucl. Phys. B352 (1991) 849.

[15] P. Fendley, A.W.W. Ludwig, and H. Saleur, Phys. Rev. B52 (1995) 8934.

[16] V. Knizhnik and A. B. Zamolodchikov, Nucl. Phys. B247 (1984) 83.

[17] D. Friedan, Z. Qiu and S. H. Shenker, Phys. Rev. Lett. 52 (1984) 1575.

[18] V. A. Fateev and A. B. Zamolodchikov, Zh. Eksp. Teor. Fiz. 89 (1986) 380 [Sov. Phys. JETP 62 (1986) 215].

[19] D. Kastor, Nucl. Phys. B280 (1987) 304; A. Capelli, Phys. Lett. B185 (1987) 82; Y. Matsuo and S. Yahikozawa, Phys. Lett. B178 (1987) 211.

[20] H. Saleur and M. Bauer, Nucl. Phys. B320 (1989) 591. 
[21] D. Bernard and A. LeClair, Nucl. Phys. B340 (1990) 721.

[22] C. Ahn, D. Bernard and A. LeClair, Nucl. Phys. B346 (1990) 409.

[23] Al. B. Zamolodchikov, Nucl. Phys. B358 (1991) 497.

[24] A. B. Zamolodchikov, Sov. Journ. Nucl. Phys. 46 (1987) 1090; A. Ludwig and J. Cardy, Nucl. Phys. B285 [FS19] (1987) 687.

[25] Al. B. Zamolodchikov, Nucl. Phys. B358 (1991) 524.

[26] Al. B. Zamolodchikov, Nucl. Phys. B366 (1991) 122.

[27] A. B. Zamolodchikov and Al. B. Zamolodchikov, Nucl. Phys. B379 (1992) 602.

[28] V. V. Bazhonov and N. Y. Reshetikhin, Int. J. Mod. Phys. A4 (1989) 115 .

[29] A. LeClair, G. Mussardo, H. Saleur and S. Skorik, Nucl. Phys. B453 [FS] (1995) 581.

[30] P. Fendley, Phys. Rev. Lett. 71 (1993) 2485.

[31] C. Ahn and W. M. Koo, Nucl. Phys. B468 (1996) 461; C. Ahn and C. K. You, J. Phys. A31 (1998) 2109.

[32] N. P. Warner, Nucl. Phys. B450 [FS] (1995) 663.

[33] C. Ahn, G. Delfino and G. Mussardo, Phys. Lett. B317 (1993) 573.

[34] C. Ahn, Nucl. Phys. B422 (1994) 449.

[35] M. J. Martins, Phys. Lett. B304 (1993) 111.

[36] C. Ahn and W.M. Koo, Nucl. Phys. B482 (1996) 675.

[37] C. Ahn, "Boundary TBA for the supersymmetric sinh-Gordon model", in preparation.

[38] P. Dorey and F. Ravanini, Nucl. Phys. B406 (1993) 708. 
hep-th/9805101 17 May 1998

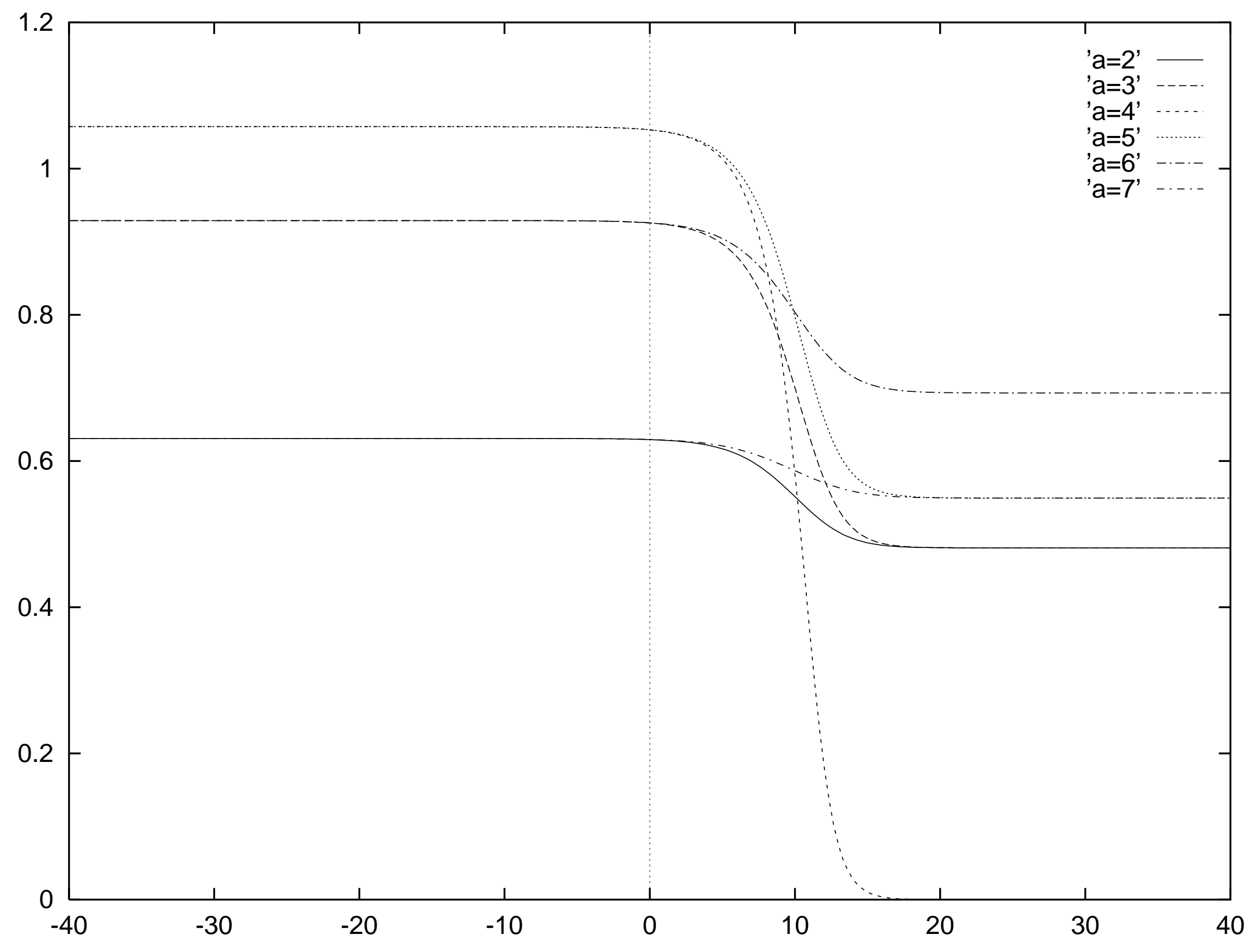


hep-th/9805101 17 May 1998

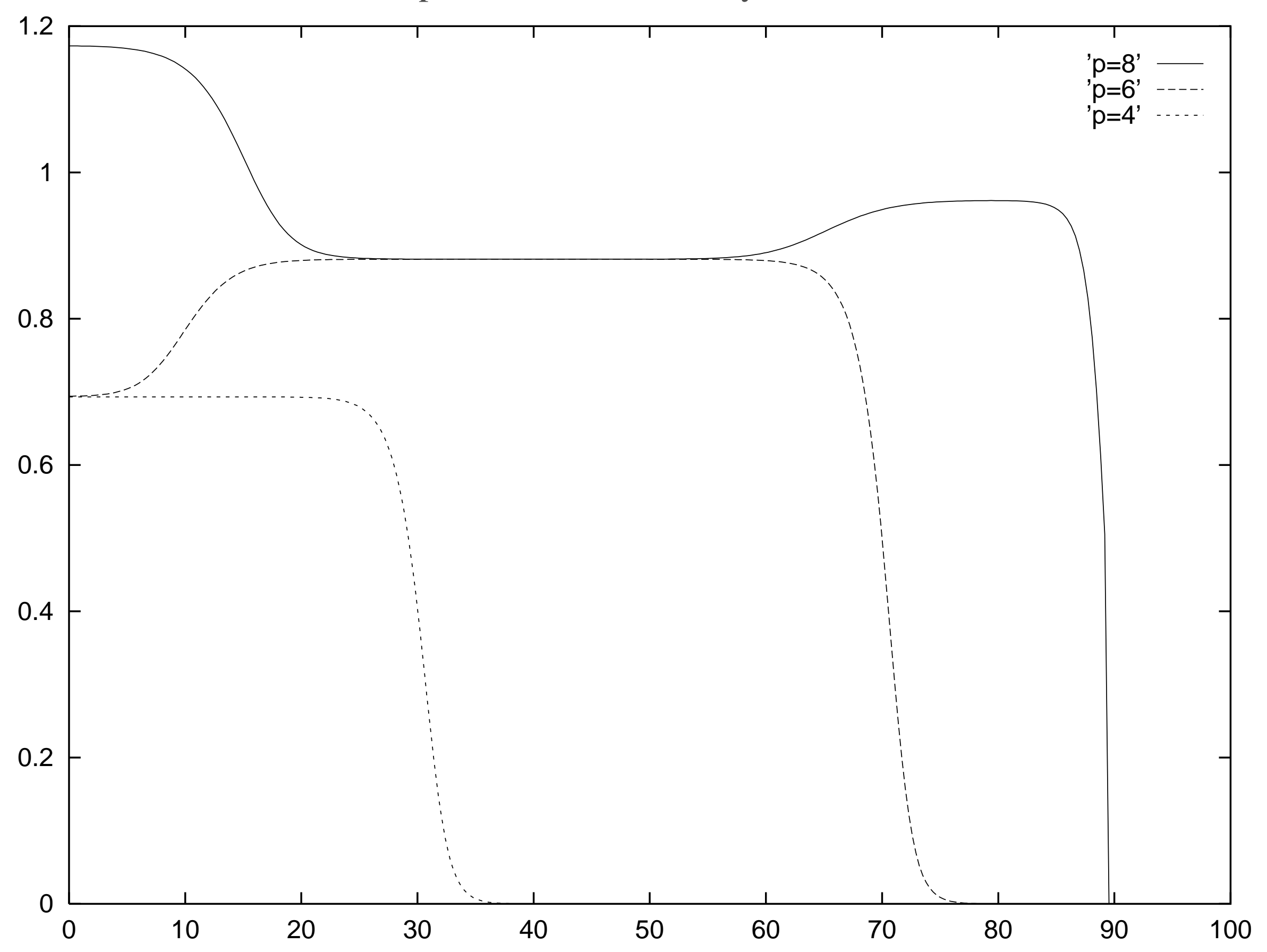


hep-th/9805101 17 May 1998

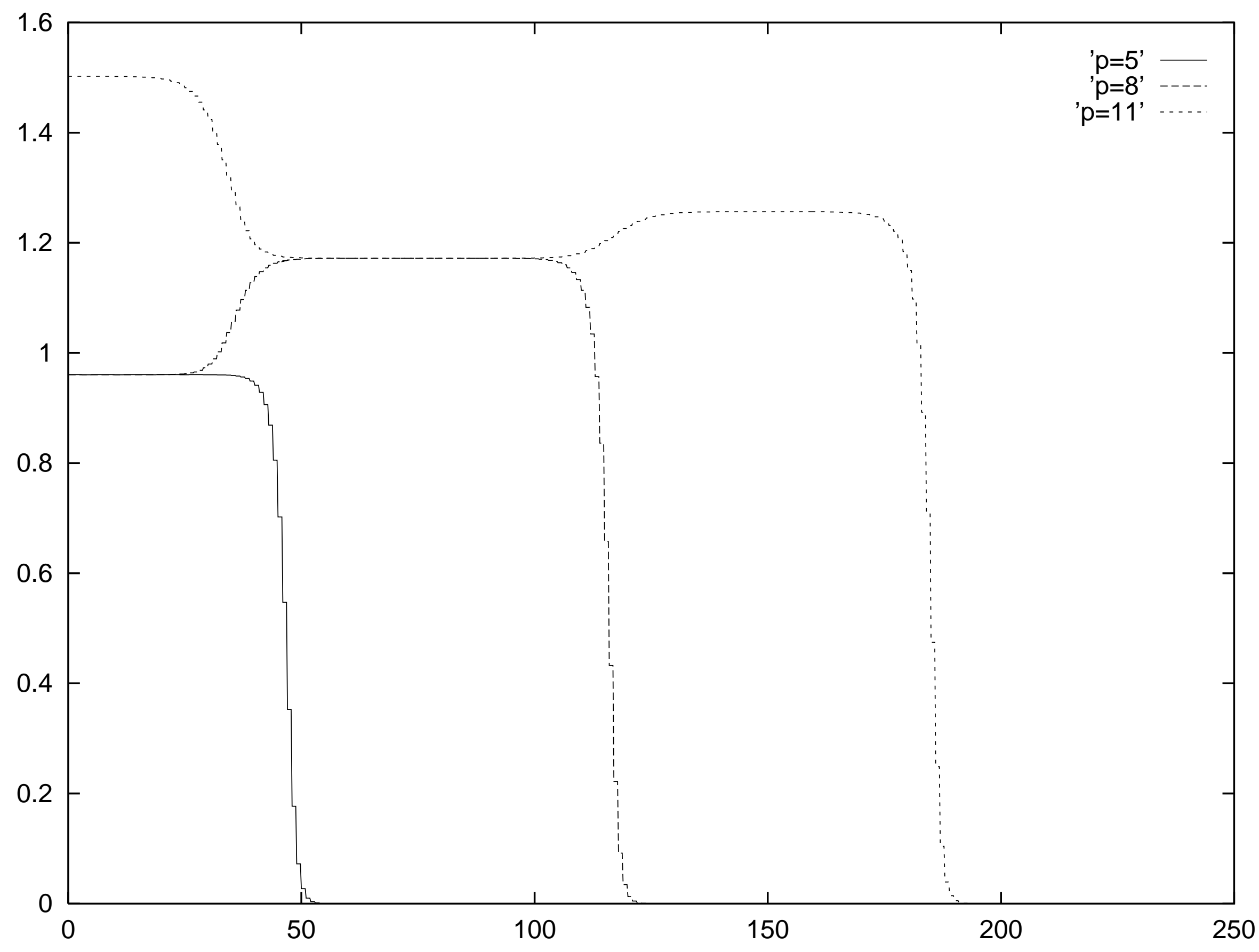

